\title{
Metástases Cutâneas e Neoplasia de Reto: Relato de Caso
}

doi: https://doi.org/10.32635/2176-9745.RBC.2020v66n3.390

\section{Cutaneous Metastasis and Rectum Neoplasia: Case Report \\ Metástasis Cutáneas y Neoplasia Rectal: Relato de Caso}

Daiane Pretto'; Fernanda Formolo²; Caroline Marsilio ${ }^{3}$

\section{RESUMO}

Introduçáo: As metástases cutâneas são as neoplasias que acometem a derme ou o tecido celular subcutâneo e podem ser um indicador de neoplasia desconhecida de mau prognóstico. Metástases cutâneas em adenocarcinoma de reto são consideradas raras. Relato do caso: Mulher de 80 anos de idade, hipertensa e diabética, apresentou dor abdominal e hematoquezia, relatando histórico familiar de câncer. Realizou colonoscopia com resultado de biópsia para adenocarcinoma. Um ano depois, recebeu terapia neoadjuvante e radioterapia concomitante por quatro meses, com boa resposta ao tratamento. Porém, relatou lesão mandibular de crescimento rápido, com o trato gastrointestinal como sítio primário de acordo com a biópsia. Em tomografias de reavaliaçáo, foram diagnosticados também vários nódulos diminutos em tecido subcutâneo de abdome inferior, mama e pulmão. Foi realizada nova biópsia dos nódulos de mama e imuno-histoquímica, procedimentos que sugeriram o reto como sítio primário das lesôes. Sendo assim, foi iniciada quimioterapia paliativa e a paciente apresentou resposta satisfatória das lesóes cutâneas logo no segundo ciclo do tratamento antineoplásico. O projeto foi aprovado pelo Comitê de Ética em Pesquisa do Hospital Pompeia sob número de parecer 311.052. Conclusáo: Este caso enfatiza que lesóes na pele de crescimento progressivo ou sem resposta após terapia convencional podem ser a primeira manifestação de câncer visceral avançado e devem ser apropriadamente investigadas. Dessa forma, fica claro que, por meio de um diagnóstico precoce, é possível mudar o prognóstico da doença e o seu tratamento.

Palavras-chave: Neoplasias Cutâneas; Neoplasias Retais; Adenocarcinoma; Metástase Neoplásica.

\section{ABSTRACT}

Introduction: Skin metastases are neoplasms that affect the dermis or subcutaneous cell tissue and may be an indicator of an unknown neoplasm with a poor prognosis. Skin metastases in rectal adenocarcinoma are considered rare. Case report: An 80-year-old woman, hypertensive and diabetic, had abdominal pain and hematochezia, reporting a family history of cancer. Colonoscopy was performed with biopsy results for adenocarcinoma. One year later, she received neoadjuvant therapy and concomitant radiation therapy for four months, with good response to treatment. However, she reported a fast-growing mandibular lesion, with the gastrointestinal tract as the primary site according to the biopsy. In reassessment CT scans, several small nodules in subcutaneous tissue of the lower abdomen, breast and lung were also diagnosed. A new biopsy of the breast nodules and immunohistochemistry were performed, procedures that suggested the rectum as the primary site of the lesions. Therefore, palliative chemotherapy was started and the patient presented a satisfactory response to skin lesions in the second cycle of antineoplastic treatment. The project was approved by the Institutional Review Board of Hospital Pompeia through report the opinion $n^{\circ}$ 311.052. Conclusion: This case emphasizes that progressive or unresponsive skin lesions after conventional therapy can be the first manifestation of advanced visceral cancer and should be properly investigated. Thus, it is clear that, through an early diagnosis, it is possible to change the prognosis of the disease and its treatment.

Key words: Skin Neoplasms; Rectal Neoplasms; Adenocarcinoma; Neoplasm Metastasis.

\section{RESUMEN}

Introducción: Las metástasis cutáneas son neoplasias que afectan la dermis o el tejido subcutáneo y pueden ser un indicador de neoplasia desconocida con mal pronóstico. Las metástasis cutáneas en el adenocarcinoma rectal se consideran raras. Relato del caso: Una mujer de 80 años, hipertensa y diabética, presentaba dolor abdominal y hematoquecia, e informaba antecedentes familiares de cáncer. La colonoscopia se realizó con resultados de biopsia para adenocarcinoma. Un año después, recibió terapia neoadyuvante y radioterapia concomitante durante cuatro meses, con buena respuesta al tratamiento. Sin embargo, informó una lesión mandibular de rápido crecimiento, con el tracto gastrointestinal como el sitio primario según la biopsia. En las tomografías de reevaluación, también se diagnosticaron varios nódulos pequeños en el tejido subcutáneo de la parte inferior del abdomen, mama y pulmón. Se realizó una nueva biopsia de los nódulos mamarios y la inmunohistoquímica, procedimientos que sugirieron el recto como el sitio primario de las lesiones. Por lo tanto, se inició la quimioterapia paliativa y el paciente presentó una respuesta satisfactoria a las lesiones cutáneas en el segundo ciclo de tratamiento antineoplásico. El proyecto fue aprobado por el Comité de Ética e Investigación del Hospital Pompéia a través del dictamen número 311.052. Conclusión: Este caso enfatiza que las lesiones cutáneas progresivas o que no responden después de la terapia convencional pueden ser la primera manifestación de cáncer visceral avanzado y deben investigarse adecuadamente. Por lo tanto, está claro que, a través de un diagnóstico temprano, es posible cambiar el pronóstico de la enfermedad y su tratamiento.

Palabras clave: Neoplasias Cutáneas; Neoplasias del Recto; Adenocarcinoma;

Metástasis de la Neoplasia.

${ }^{1}$ Universidade de Caxias do Sul (UCS). Hospital Pompeia de Caxias do Sul. Caxias do Sul (RS), Brasil. Orcid iD: https://orcid.org/0000-0001-5852-2184 ${ }^{2}$ UCS. Centro de Pesquisa Clínica do Hospital Pompeia de Caxias do Sul. Centro Universitário da Serra Gaúcha (FSG). Caxias do Sul (RS), Brasil. Orcid iD: https://orcid. org/0000-0002-2030-9860

${ }^{3}$ Centro de Pesquisa Clínica do Hospital Pompeia de Caxias do Sul. FSG. Caxias do Sul (RS), Brasil. Orcid iD: https://orcid.org/0000-0003-4721-5503 Endereço para correspondência: Fernanda Formolo. Av. Júlio de Castilhos, 2163 - Centro. Caxias do Sul (RS), Brasil. CEP 95010-005. E-mail: fernanda.formolo@pompeia.org.br 


\section{INTRODUÇÃO}

Metástase é definida como uma lesão neoplásica que se origina a partir de outra neoplasia, com a qual não está mais em continuidade ${ }^{1}$. A metástase cutânea, por sua vez, é caracterizada como sendo a neoplasia que envolve a derme ou o tecido celular subcutâneo ${ }^{2}$, e ocorre somente em $0,7 \%$ a $10,4 \%$ de todos os pacientes com câncer ${ }^{3}$. Essas lesōes cutâneas, por sua vez, possuem uma ampla variedade de aparências, muitas vezes simulando lesôes benignas ${ }^{4}$. Na maioria dos casos, ocorre tardiamente no curso da doença, mas pode ser o sinal de apresentação de uma neoplasia desconhecida ${ }^{5}$. Assim, um alto índice de suspeita clínica é necessário para o diagnóstico das lesôes metastáticas da pele, pois, quando presente, a metástase cutânea é fator isolado de mau prognóstico oncológico ${ }^{6}$.

O projeto foi aprovado pelo Comitê de Ética em Pesquisa do Hospital Pompeia sob o número de parecer 311.052 .

\section{RELATO DO CASO}

Paciente do sexo feminino, com 80 anos de idade ao diagnóstico, relatou aparecimento de hematoquezia associada à dor abdominal crescente no início do ano de 2017. Esta apresenta como comorbidades hipertensáo arterial e diabetes mellitus náo insulinodependente, relata ter cessado o uso de tabaco há 20 anos e possuir histórico familiar de câncer (filho com câncer de reto, uma irmã com câncer de mama, outra de estômago e um irmão com neoplasia renal). Neste mesmo ano, realizou colonoscopia, a qual evidenciou lesão infiltrativa estenosante e friável de reto, revelando em biópsia um adenocarcinoma. Posteriormente, realizou-se estadiamento e revelou-se ausência de doença a distância (cT3cN1M0). Cerca de um ano após o diagnóstico, foi realizada uma colostomia, a qual evidenciou necessidade do uso de neoadjuvância com fluorouracil e radioterapia concomitante durante quatro meses. Após esse período, realizou-se nova colonoscopia, constatando boa resposta ao tratamento. Contudo, quatro meses após, a paciente referiu notar lesão submandibular esquerda de crescimento rápido (Figura 1). Biópsia incisional dessa lesão e imuno-histoquímica foram realizadas, favorecendo o trato gastrointestinal como sítio primário. Em tomografias de reavaliação, foram diagnosticados vários nódulos diminutos em tecido subcutâneo de abdome inferior, sendo um localizado em dorso, com dimensóes de 2,6 x 2,2 cm, e outro situado na mama direita, com 2,9 × 2,9 cm, assim como pequenos nódulos pulmonares esparsos. Novamente, foram realizados os procedimentos de biópsia dos nódulos de mama e imuno-histoquímica, os quais sugeriram

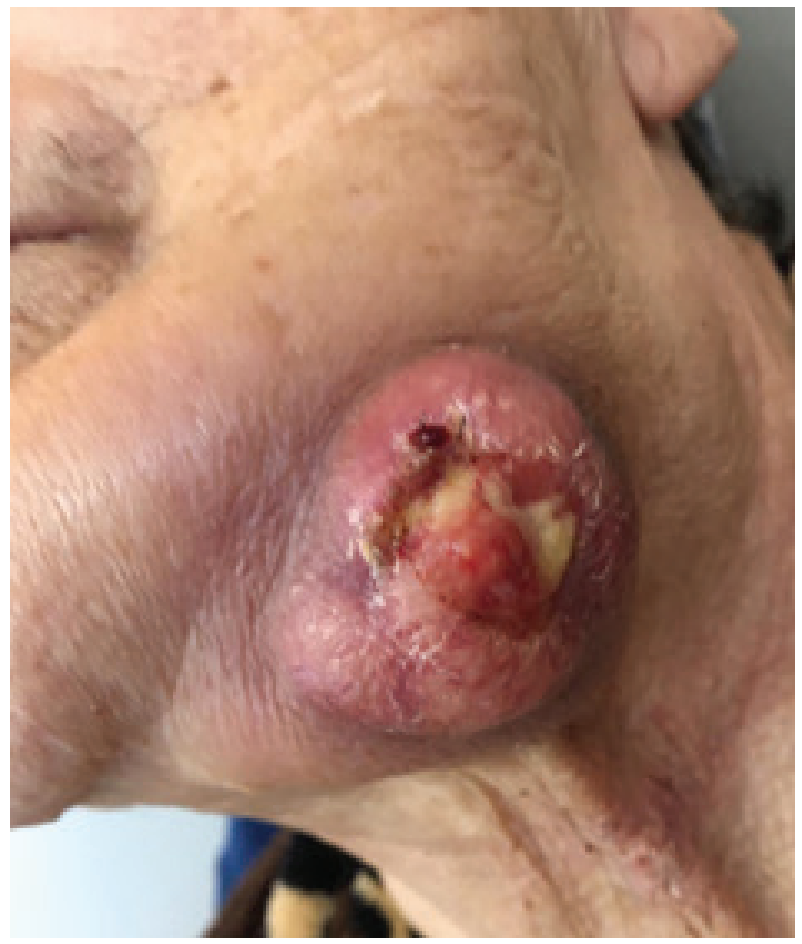

Figura 1. Lesão submandibular esquerda - Metástase cutânea

Fonte: Acervo do autor.

o reto como sítio primário das lesóes. Sendo assim, a quimioterapia paliativa teve início quatro meses após o surgimento da lesão submandibular.

A paciente apresentou resposta satisfatória das lesôes cutâneas logo no segundo ciclo do tratamento antineoplásico. Ao mês de redação deste relato, dezembro de 2019, a paciente está há 20 meses do diagnóstico da neoplasia e há 15 meses do diagnóstico da metástase cutânea, segue realizando quimioterapia paliativa e mantendo boa resposta ao tratamento, com qualidade de vida preservada.

\section{DISCUSSÃO}

O câncer de cólon e reto é a terceira neoplasia mais comumente diagnosticada no mundo, com um valor estimado de 1,8 milhão de novos casos em $2018^{7,8}$, e de caráter multifatorial, sendo assim de etiologia influenciada por fatores genéticos, ambientais e relacionados ao estilo de vida9 ${ }^{9}$. Os fatores hereditários, como o histórico familiar de câncer e as doenças inflamatórias do intestino, representam apenas uma pequena proporção da variação observada na carga global da doença ${ }^{8,10}$. Os fatores de risco ligados ao estilo de vida são modificáveis e incluem o consumo de bebidas alcoólicas, a baixa ingestâo de frutas e vegetais, o alto consumo de carnes vermelhas e de alimentos processados, a obesidade, o tabagismo e a inatividade física ${ }^{7,811,12}$. 
No Brasil, em 2017, ocorreram 9.207 óbitos por câncer de cólon e reto em homens e 9.660 em mulheres ${ }^{7,8,13}$, e, para cada ano do triênio 2020-2022, estimam-se, no país, 20.520 casos novos de câncer de cólon e reto em homens e 20.470 em mulheres. Esses valores correspondem a um risco estimado de 19,64 casos novos a cada 100 mil homens e 19,03 para cada 100 mil mulheres. Sendo considerada a terceira neoplasia mais frequente em homens e a segunda entre as mulheres, exceto câncer de pele não melanoma, no contexto nacional ${ }^{8,14}$.

A ocorrência de metástase cutânea como primeira manifestação de doença metastática é rara e indica uma doença em estágio avançado ${ }^{15}$ e de prognóstico pobre. Cerca de $60 \%$ das metástases cutâneas são de adenocarcinomas, sendo os sítios primários que, com maior frequência, produzem metástase os de mama, do trato gastrointestinal e pulmonares ${ }^{16}$ e, mais raramente, as neoplasias de pâncreas, glândula parótida, amígdalas, melanoma, ovário e útero ${ }^{17}$. As que indicam pior prognóstico ocorrem principalmente em doentes com câncer de ovário, pulmão e trato digestivo superior. A maioria ocorre em locais próximos ao tumor primário e a apresentação mais comum são nódulos indolores e firmes ${ }^{6}$.

Em estudo realizado com 209 pacientes com metástases, a região mais acometida foi a porção anterior do tórax, $(31,94 \%)$, seguida da região abdominal $(20,37 \%)$ e do couro cabeludo (14,81\%). Sendo o câncer de mama o principal responsável por metástases cutâneas em mulheres $(63,19 \%)$ e os pulmóes $(33,84 \%)$ nos homens ${ }^{4}$. Apesar das técnicas de imagem e imuno-histoquímica, a localização do tumor primário não pode ser determinada em 5\% a 10\% dos $\operatorname{casos}^{4,18}$. Em virtude dos avanços na terapia do câncer, a expectativa de vida dos pacientes com metástases cutâneas aumentou, mas essas metástases continuam a ser um fator determinante de mau prognóstico ${ }^{19}$.

\section{CONCLUSÃO}

Este caso enfatiza que lesóes na pele de aparecimento recente, que mostram crescimento progressivo ou que náo se curam após terapia convencional, podem ser a primeira manifestação de câncer visceral avançado e devem ser apropriadamente investigadas. Dessa forma, fica claro que, por meio de um diagnóstico precoce, é possível mudar o prognóstico da doença e o seu tratamento. $\mathrm{O}$ profissional de saúde deve estar atento, frente a esses sinais clínicos, elevando a suspeita, valorizando a anamnese e direcionando a investigação. Quanto mais precoce o diagnóstico, melhor será o resultado do tratamento. Portanto, um exame clínico completo deve ser sempre realizado, e todas as nodulaçóes com aumento persistente ou placas endurecidas devem ser submetidas à biópsia para descartar o diagnóstico de metástase cutânea. Apesar do prognóstico reservado da paciente, esta permanece com boa resposta à quimioterapia e qualidade de vida preservada. Visto que produçóes sobre a temática são escassas, a relevância do presente estudo se encontra no fato de que a explanação de um caso considerado raro, e suas respectivas condutas, possui potencial de guiar outros profissionais em sua prática clínica quando confrontados com situaçóes semelhantes.

\section{CONTRIBUIÇÕES}

Daiane Pretto contribuiu substancialmente na concepção e planejamento do estudo, na obtenção, análise e interpretação dos dados, assim como na redação e revisão crítica. Fernanda Formolo e Caroline Marsilio contribuíram substancialmente na redação e revisão crítica. Todas as autoras aprovaram a versão final a ser publicada.

\section{DECLARAÇÃO DE CONFLITO DE INTERESSES}

Nada a declarar.

\section{FONTES DE FINANCIAMENTO}

Não há.

\section{REFERÊNCIAS}

1. Bittencourt MJS, Carvalho AH, Nascimento BAM, et al. Metástase cutânea de câncer de mama diagnosticado 13 anos antes. An Bras Dermatol. 2015;90(3 Supl 1):S132-5.

2. Caldas FAA, Curtis JAG, Baldelin TAR, et al. Recidivas cutâneas de tumores mamários: formas de apresentação e diagnóstico diferencial. Rev Imagem [Internet]. 2016 [acesso 2019 ago 1];28(3):197-201. Disponível em: http:// rpcadm.hospitalmoinhos.org.br/Arquivos/3354dd71482e-4dde-990c-ff73cb324081.pdf

3. De Giorgi V, Grazzini M, Alfaioli B, et al. Cutaneous manifestations of breast carcinoma. Dermatol Ther. 2010;23(6):581-9. doi: https://doi.org/10.1111/j.15298019.2010.01365.x

4. Sittart JAS, Senise M. Metastases cutaneas de carcinomas internos: revisao de 45 anos. An Bras Dermatol. 2013;88(4):549-552.

5. Krathen RA, Orengo IF, Rosen T. Cutaneous metastasis: a meta-analysis of data. South Med J. 2003;96(2):164-7. doi: https://doi.org/10.1097/01.SMJ.0000053676.73249.E5

6. Wong CYW, Helm MA, Kalb RE, et al. The presentation, pathology, and current management strategies of 
cutaneous metastasis. N Am J Med Sci. 2013;5(9):499504. doi: https://doi.org/10.4103/1947-2714.118918

7. Siegel RL, Torre LA, Soerjomataram I, et al. Global patterns and trends in colorectal cancer incidence in young adults. Gut, 2019;68(12):2179-2185. doi: https:// doi.org/10.1136/gutjnl-2019-319511

8. Instituto Nacional de Câncer José Alencar Gomes da Silva. Estimativa 2020: incidência de cancer no Brasil [Internet]. Rio de Janeiro: INCA; 2019 [acesso 2019 ago 1]. Disponível em: https://www.inca.gov.br/sites/ ufu.sti.inca.local/files/media/document/estimativa-2020incidencia-de-cancer-no-brasil.pdf

9. Boyle P, Leon ME. Epidemiology of colorectal cancer. Br Med Bull. 2002;64(1):1-25. doi: https://doi. org/10.1093/bmb/64.1.1

10. Arnold M, Sierra MS, Laversanne M, et al. Global patterns and trends in colorectal cancer incidence and mortality. Gut. 2017;66(4):683-91. doi: https://doi. org/10.1136/gutjnl-2015-310912

11. Bouvard V, Loomis D, Guyton KZ, et al. Carcinogenicity of consumption of red and processed meat. Lancet. Oncol. 2015;16(16):1599-600. doi: https://doi. org/10.1016/S1470-2045(15)00444-1

12. Walter V, Jansen L, Hoffmeister M, et al. Smoking and survival of colorectal cancer patients: systematic review and meta-analysis. Ann Oncol. 2014;25(8):1517-25. doi: https://doi.org/10.1093/annonc/mdu040

13. SIM: Sistema de Informação sobre Mortalidade [Internet]. Brasília, DF: Ministério da Saúde. c2008 [acesso 2019 maio 28]. Disponível em: http://tabnet. datasus.gov.br/cgi/tabcgi.exe?sim/cnv/obt10uf.def

14. Instituto Nacional de Câncer José Alencar Gomes da Silva [Internet]. Rio de Janeiro: INCA; [data desconhecida]. Estimativa 2020: síntese de resultados e comentários; 2020 [atualizado 2020 maio 12; acesso em 2020 maio 12]. Disponível em: http://www1.inca.gov.br/ estimativa/2018/sintese-de-resultados-comentarios.asp

15. Fyrmpas G, Barbetakis N, Efstathiou A, et al. Cutaneous metastasis to the face from colon adenocarcinoma. Case report. Int Semin Surg Oncol. 2006;3:2. doi: https:// doi.org/10.1186/1477-7800-3-2

16. Weimann ETS, Botero EB, Mendes C, et al. Metástase cutânea como primeira manifestação de neoplasia maligna oculta de mama - Relato de caso. An Bras Dermatol. 2016;91(5 Supl 1):S105-7.

17. Araujo $A B$, Barbosa $A B$, Bellé Júnior $C$, et al. $A$ importância do diagnóstico precoce de metástases cutâneas de neoplasias internas. Rev UNINGÁ Review [Internet]. 2013 [acesso 2019 ago 5];15(1):41-43. Disponível em: http://revista.uninga.br/index.php/ uningareviews/article/download/723/371/

18. van den Hurk CJG, Eckel R, van de Poll-Franse LV, et al. Unfavourable pattern of metastases in $\mathrm{M} 0$ breast cancer patients during 1978-2008: a population-based analysis of the Munich Cancer Registry. Breast Cancer Res Treat. 2011;128(3):795-805. doi: https://doi.org/10.1007/ s10549-011-1372-y

19. Junqueira ALA, Corbett AMF, Oliveira Filho J, et al. Metástase cutânea de adenocarcinoma do trato gastrointestinal de sítio primário desconhecido. An Bras Dermatol. 2015;90(4):564-6. 